\title{
A REMARK ON A TRACE PALEY-WIENER THEOREM
}

\author{
GORAN MUIĆ
}

\begin{abstract}
In this paper we prove a version of a trace Paley-Wiener theorem for tempered representations of a reductive $p$-adic group. This is applied to complete certain investigation of Shahidi on the proof that a Plancherel measure is invariant of a $L$-packet of discrete series.
\end{abstract}

\section{INTRODUCTION}

Let $G$ be a reductive $p$-adic group. Let $\operatorname{Rep}(G)$ be the category of smooth admissible complex representations of $G$ of finite length, and let $R(G)$ be the corresponding Grothendieck group. We write $\Psi(G)$ (resp., $\Psi^{u}(G)$ ) for the group of (resp., unitary) unramified characters of $G$. The group $\Psi(G)$ has a structure of an algebraic variety (a complex tours). The corresponding algebra of regular functions $\mathbb{C}[\Psi(G)]$ is generated by evaluations on elements of $G$ as a $\mathbb{C}$-algebra. The subgroup $\Psi^{u}(G)$ is Zariski dense in $\Psi(G)$. We say that a complex function is regular on $\Psi^{u}(G)$ if it is a restriction of a regular function on $\Psi(G)$. We observe that the restriction map from $\mathbb{C}[\Psi(G)]$ into functions on $\Psi^{u}(G)$ is injective since $\Psi^{u}(G)$ is Zariski dense in $\Psi(G)$.

We fix a minimal parabolic subgroup $P_{0}$, its Levi decomposition $P_{0}=$ $M_{0} U_{0}$, and, as usual related to these choices, we fix a set of standard parabolic subgroups $P=M U$, where $M_{0} \subset M, P=M P_{0}$. Since the standard parabolic subgroup is determined by the choice of Levi subgroup, the normalized parabolic induction $\operatorname{Ind}_{P}^{G}(\sigma)$, where $\sigma$ is a smooth representation of $M$, we write as usual $i_{G M}(\sigma)$.

In [3], Bernstein, Deligne, and Kazhdan proved a trace Paley-Wiener theorem for category $\operatorname{Rep}(G)$. We consider a full subcategory $\operatorname{Rep}_{t}(G)$ of $\operatorname{Rep}(G)$ consisting of representations having all irreducible subquotients tempered. Let $R_{t}(G)$ be the corresponding Grothendieck group. We write $R_{t}^{i}(G)$ for the subgroup of $R_{t}(G)$ generated by $i_{G M}(\sigma)$, where $M$ ranges over all standard Levi subgroups of $G$ (including $G$ ), and $\sigma$ ranges over a set of square-integrable modulo center irreducible representations of $M$. We warn the reader that this notion is not an analogue of the notion of strictly induced modules from ([3], 3.1). An analogue would be the subgroup of $R_{t}(G)$ generated by $i_{G M}(\tau)$, where where $M$ ranges over all proper standard Levi

2010 Mathematics Subject Classification. 11E70, 22E50.

Key words and phrases. Paley-Wiener theorem, admissible representations, reductive $p$-adic groups.

The author acknowledges Croatian Science Foundation grant no. 9364. 
subgroups of $G$, and $\tau$ ranges over irreducible tempered representations of $M$. But this is not useful for us in the present paper.

The main result of the present paper is the following version of a trace Paley-Wiener theorem:

Theorem 1-1. Let $f: R_{t}(G) \longrightarrow \mathbb{C}$ be a $\mathbb{Z}$-linear form such that the following hold:

(i) There exists an open compact subgroup $K \subset G$ which dominates $f$ (i.e., $f$ is non-zero only on those irreducible tempered representations which have a non-trivial space of $K$-invariant vectors),

(ii) For each standard maximal Levi subgroup $M$, or $M=G$, and a square-integrable modulo center representation $\sigma$ of $M$, the function $\psi \mapsto f\left(i_{G M}(\psi \sigma)\right)$ is regular on $\Psi^{u}(M)$, and for any other proper standard Levi subgroup $N$, and a square-integrable modulo center representation $\tau$ of $N$, we have $f\left(i_{G N}(\tau)\right)=0$.

Then, there exists $F \in C_{c}^{\infty}(G)$ such that

$$
f(\pi)=\operatorname{tr}(\pi(F)), \quad \text { for all } \pi \in R_{t}^{i}(G) .
$$

Theorem 1-1 is a proved by reduction to the main result of [3] using Harish-Chandra theory of tempered representations [13] and some standard considerations related to the Langlands classification ([11, Chapter VII). The proof is given in Section 3. It is a consequence of its effective version given by Proposition 3-4, Proposition 3-4 constructs a correct function needed in the proof of (12], Proposition 9.32$)$ ) in the case when $M$ (see notation there) is a Levi of a maximal parabolic subgroup. We remark that since Plancherel factors are multiplicative, it is enough to prove ([12], Proposition 9.3 2)) for a maximal Levi subgroup.

I would like to than Gordan Savin for turning my attention to this question. A draft of the paper was written while the author visited the Hong Kong University of Science and Technology in January of 2018. The author would like to thank A. Moy and the Hong Kong University of Science and Technology for their hospitality.

\section{Preliminaries}

We continue with the notation introduced in the introduction. Let $M$ be a standard Levi subgroup. Then, we write $\Psi(M)^{r}$ for the group of all unramified characters $\psi$ which are $\mathbb{R}_{>0}$-valued. As we stated in the introduction, every standard Levi subgroup $M$ determine unique standard parabolic subgroup, say $P$. We denote by $\Psi(M)^{r,+}$ the set of all characters from $\Psi(M)^{r}$ which correspond to the points of the (open) Weyl chamber determined by the roots of the split component of $M$ which belong to the 
unipotent radical of $P$ in the usual description of unramified characters (see for example [10, Section 2). If $M=G$, then $\Psi(M)^{r,+}=\Psi(M)^{r}$.

For a standard Levi subgroup $M$, an irreducible tempered representation $\pi$ of $M$, and $\psi \in \Psi(M)^{r,+}$, the module $i_{G M}(\psi \pi)$ is called a standard module; it has a unique (Langlands quotient) $L\left(i_{G M}(\psi \pi)\right)$. The condition is empty if $M=G$. By the Langlands classification ([11], Theorem VII.4.2), every irreducible representation can be expressed in the form $L\left(i_{G M}(\psi \pi)\right)$ for unique such datum $(M, \pi, \psi)$. The following standard result will be used in the proof:

Lemma 2-1. The standard modules of $G$ form a $\mathbb{Z}$-basis of $R(G)$.

Proof. As in ([8], Proposition 1).

In analogy with $([3,2.1)$, we make the following definitions.

Let $\sigma \in \operatorname{Irr}(M)$ where $M$ is a standard Levi subgroup of $G$. We define the usual affine variety attached to $\sigma$

$$
\operatorname{Irr}(M) \supset D(\sigma)=\Psi(M) \sigma=\Psi(M) / \operatorname{Stab}_{\Psi(M)}(\sigma),
$$

where $\operatorname{Stab}_{\Psi(M)}(\sigma)$ is a finite group consisting of all $\psi \in \Psi(M)$ such that $\psi \sigma \simeq \sigma$.

If $A$ is a maximal split torus in the centre of $M$, then the restriction map $\Psi(M) \rightarrow \Psi(A)$ is surjective, and the kernel is a finite group. Therefore, by considering the restriction to $A$ we find that

$$
\operatorname{Stab}_{\Psi^{u}(M)}(\sigma)=\operatorname{Stab}_{\Psi(M)}(\sigma) .
$$

So, we may consider

$$
D^{u}(\sigma) \stackrel{\text { def }}{=} \Psi^{u}(M) / \operatorname{Stab}_{\Psi^{u}(M)}(\sigma) \subset D(\sigma) .
$$

It is easy to see that $D^{u}(\sigma)$ is Zariski dense in $D(\sigma)$.

The action of the Weyl group

$$
W(M)=N_{G}(M) / M
$$

on $\Psi(M)$ is algebraic. Moreover, $w \in W(M)$ transforms $\operatorname{Stab}_{\Psi(M)}(\sigma)$ onto $S_{t a b_{\Psi}(M)}(w(\sigma))$. So that it maps $D(\sigma)$ (resp., $\left.D^{u}(\sigma)\right)$ onto $D(w(\sigma))$ (resp., $\left.D^{u}(w(\sigma))\right)$.

Put $D=D(\sigma)$ and $D^{u}=D^{u}(\sigma)$. As usually, we consider the group $W(D)$ of all $w \in W(M)$ such that there exists $\psi_{w} \in \Psi(M)$ such that

$$
w(\sigma) \simeq \psi_{w} \sigma .
$$


The character $\psi_{w}$ is determined uniquely modulo $\operatorname{Stab}_{\Psi(M)}(\sigma)$. The group $W(D)$ acts on the affine variety $D=\Psi(M) / \operatorname{Stab}_{\Psi(M)}(\sigma)$ as follows:

$$
w \cdot \psi \operatorname{Stab}_{\Psi(M)}(\sigma)=\psi_{w} w(\psi) \operatorname{Stab}_{\Psi(M)}(\sigma) .
$$

The resulting orbit space

$$
D / W(D)
$$

is again an affine variety with algebra of regular functions given as usual

$$
\mathbb{C}[D / W(D)]=\mathbb{C}[D]^{W(D)} .
$$

One can construct a regular function $D / W(D)$ in the following way:

Lemma 2-4. Let $F \in C_{c}^{\infty}(G)$. Then, the function $\psi \longmapsto \operatorname{tr}\left(i_{G M}(\psi \sigma)(F)\right)$ is a regular function on $D / W(D)$.

Proof. It is standard that this function is regular on $D$. We show that it is $W(D)$-invariant. Let $w \in W(D)$. By ([3], Lemma 5.4 (iii)), we have

$$
\operatorname{tr}\left(i_{G M}(\psi \sigma)(F)\right)=\operatorname{tr}\left(i_{G M}(w(\psi \sigma))(F)\right)
$$

which completes the proof.

The above explicit description shows that analogously defined group $W\left(D^{u}\right)$ is a subgroup of $W(D)$. In fact, we have the following lemma:

Lemma 2-5. Assume that the central character $\omega_{\sigma}: A \longrightarrow \mathbb{C}^{\times}$of $\sigma$ is unitary. Then, $W\left(D^{u}\right)=W(D)$. Moreover, $D^{u} / W(D)$ is Zariski dense in $D / W(D)$.

Proof. As we remarked above, it is always $W\left(D^{u}\right) \subset W(D)$. Conversely, if $w \in W(D)$, then $w(\sigma) \simeq \psi_{w} \sigma$ by (2-2). Considering central characters, we find that

$$
\omega_{w(\sigma)}=\left(\psi_{w} \mid A\right) \omega_{\sigma} .
$$

This implies that $\left.\psi_{w}\right|_{A}$ is an unitary character. By the standard description of unramified characters of $M$, and its relation to unramified characters of $A$, this implies that $\psi_{w} \in \Psi^{u}(M)$ (see [10], Section 2). Hence, $w \in W\left(D^{u}\right)$. This completes the proof that $W\left(D^{u}\right)=W(D)$. The remaining claim is obvious from above considerations.

The following lemma is a fundamental result of Harish-Chandra:

Lemma 2-6. Assume that $M$ and $N$ are standard Levi subgroups of $G$, and $\sigma$ and $\tau$ are square-integrable modulo center representations of $M$ and $N$, respectively. Then, $i_{G M}(\sigma)$ and $i_{G N}(\tau)$ have a common irreducible subrepresentation if and only if there exists $w \in G$ such that $N=w M w^{-1}$ 
and $\tau \simeq w(\sigma)$, where $w(\sigma)$ is defined by $w(\sigma)(n)=\sigma\left(w^{-1} n w\right), n \in N$. Moreover, if there exists $w \in G$ such that $N=w M w^{-1}$, then $i_{G M}(\sigma)$ and $i_{G M}(w(\sigma))$ are isomorphic, and in particular equal in $R_{t}(G)$.

Proof. [13.

Motivated by ([3], 2.1), we proceed as follows. By the standard theory of tempered irreducible representations due to Harish-Chandra (see [13]), for an irreducible tempered representation $\pi \in \operatorname{Irr}(G)$, there exists a standard Levi subgroup $M$ and a square-integrable modulo center representation $\sigma$ of $M$ such that $\pi \hookrightarrow i_{G M}(\sigma)$. The pair is $(M, \sigma)$ is unique up to a conjugation (see Lemma 2-6) . We call the equivalence class $[M, \sigma]$ under conjugation of the pair $(M, \sigma)$ the $t$-infinitesimal character of $\pi$. The set of equivalence of such pairs we denote by $\Theta_{t}(G)$.

For a pair $(M, \sigma)$, we define a natural map $\Psi^{u}(M) \longrightarrow \Theta_{t}(G)$ given by

$$
\psi \longmapsto[M, \psi \sigma] .
$$

The image is called a connected component of $\Theta_{t}(G)$. We denote it by $\Theta_{t}(M, \sigma)$. This map induces a bijection which enable us to identify

$$
\Theta_{t}(M, \sigma)=D^{u}(\sigma) / W(D(\sigma)) .
$$

Thus, in view of Lemma 2-5, we may consider

$$
\Theta_{t}(M, \sigma) \subset D(\sigma) / W(D(\sigma)) .
$$

This realizes $\Theta_{t}(M, \sigma)$ as a Zariski dense subset of the affine variety $D(\sigma) / W(D(\sigma))$.

As in $([3,2.1)$, we can decompose

$$
R_{t}(G)=\oplus_{\theta} R_{t}(G)(\theta),
$$

where $\theta$ ranges over connected components of $\Theta_{t}(G)$. Here

$$
R_{t}(G)(\theta)
$$

is generated with all tempered irreducible representations which $t$-infinitesimal characters belong to $\theta$. We denote by $1_{\theta}$ the projector

$$
R_{t}(G) \rightarrow R_{t}(G)(\theta)
$$

for all $\theta \in \Theta_{t}(G)$.

We end this section with an analogue for $\operatorname{Rep}_{t}(G)$ of the Decomposition theorem for the category of all smooth complex representations of $G$ (see [3] $2.3 ;$ [1, 2.10). 
Lemma 2-8. Let $K \subset G$ be an open compact subgroup. Then, there exists a finite set $T_{K}$ consisting of connected components in $\Theta_{t}(G)$ such that for each irreducible tempered representation $\pi \in \operatorname{Rep}_{t}(G)$, having non-zero space of $K$-invariants, there exists $\theta \in T_{k}$ such that $\pi \in R_{t}(G)(\theta)$.

Proof. By the Decomposition theorem (see [3, 2.3), there exists a finite set, say $S$, of pairs $(N, \rho)$, where $N$ is a standard Levi subgroup of $G$, and $\rho$ are irreducible supercuspidal representations, such that for every irreducible representation $\pi$ of $G$, having non-zero space of $K$-invariants, there exists $(N, \rho) \in S$, and an unramified character $\chi$ such that $\pi$ is a subqoutient of $i_{G, N}(\chi \rho)$.

Now, assume that $\pi$ is as in the statement of the lemma. Then, there exists a standard Levi subgroup $M$ and a square-integrable modulo center $\sigma$ of $M$ such that $\pi \hookrightarrow i_{G M}(\sigma)$. Moreover, there exists a standard Levi subgroup $M^{\prime}$ of $M$ (and of $G$ ), and a supercuspidal irreducible representation $\rho^{\prime}$ such that $\sigma$ is an irreducible subquotient of $i_{M, M^{\prime}}\left(\rho^{\prime}\right)$. By induction in stages, $\pi$ must be a subquotient of $i_{G, M^{\prime}}\left(\rho^{\prime}\right)$. By standard theory of induced representation [5], the pair $\left(M^{\prime}, \rho^{\prime}\right)$ must be $G$-conjugate to the one in $S$. Thus, we may assume that $\left(M^{\prime}, \rho^{\prime}\right) \in S$ already.

Thus, it is enough to prove that given $(N, \rho) \in S$ and given standard Levi subgroup $M$ of $G$ such that $N \subset M$, there are finitely many of $\Psi^{u}(M)-$ orbits of square-integrable modulo center representations of $M$ such they are subqoutients of the induced representations in the family $i_{M, N}(\chi \rho)$ parameterized by $\chi \in \Psi(N)$. But that is easy. We can select sufficiently small open compact subgroup $L \subset M$ such that every irreducible representation that appear as a subqoutient of $i_{M, N}(\chi \rho)$ for some $\chi \in \Psi(N)$ has a non-zero space of $L$-invariants.

Hence, we need to prove that there are finitely many of $\Psi^{u}(M)$-orbits of square-integrable modulo center representations of $M$ having a non-zero space of $L$-invariants. This is proved in [13] (see (iii) in the introduction of [13]).

\section{Proof of Theorem 1-1}

We begin the proof of Theorem 1-1] with the following lemma:

Lemma 3-1. Let $f$ be as in the statement of Theorem 1-1. Then, there exists a finite set $T_{f}$ consisting of connected components in $\Theta_{t}(G)$ such that for each irreducible tempered representation $\pi \in \operatorname{Rep}_{t}(G)$ such that $f(\pi) \neq 0$ there exists $\theta \in T_{f}$ such that $\pi \in R_{t}(G)(\theta)$.

Proof. This follows from the assumption (i) in Theorem 1-1 combined with Lemma 2-8. 
By Lemma 3-1, we can decompose $f$ into $\mathbb{Z}$-linear forms $f_{\Theta}: R_{t}(G) \longrightarrow$ $\mathbb{C}, \theta \in T_{f}$,

$$
f=\sum_{\theta \in T_{f}} f_{\theta},
$$

where $f_{\theta}$ is defined as follows (see (2-7)):

$$
f_{\theta}=f \circ 1_{\theta} \text {. }
$$

Obviously, each $f_{\theta}$ satisfies the assumptions analogous to (i) and (ii) in Theorem 1-1.

Hence, in what follows we may assume that $f=f_{\theta}$ for some $\theta \in \Theta_{t}(G)$. By the assumption (ii) of Theorem 1-1, we may assume that $\theta$ has the form $\theta=\Theta_{t}(M, \sigma)$, where $M$ is a standard maximal Levi subgroup of $G$, or $M=G$, and $\sigma$ is a $\sigma$ is a square-integrable modulo center representation of $M$. We observe that

$$
\psi \in \Psi^{u}(M) \longmapsto f\left(i_{G M}(\psi \sigma)\right)
$$

is a regular function by the assumption (ii) of Theorem 1-1, Thus, by definition this means, that it is restriction of a regular function, say $a$ of on the affine variety $\Psi(M)$. By Lemma 2-6, we have

$$
a \in \mathbb{C}[D]^{W(D)},
$$

where

$$
D=\Psi(M) / \operatorname{Stab}_{\Psi(M)}(\sigma) .
$$

We refer to previous section for the notation.

Now, the following proposition completes the proof of the theorem.

Proposition 3-4. Let $M$ be a standard maximal Levi subgroup of $G$, or $M=G$. Assume that $\sigma$ is a square-integrable modulo center representation of $M$. We define $D$ by (3-3), and let a be any function in $\mathbb{C}[D]^{W(D)}$. Then, there exists $F \in C_{c}^{\infty}(G)$ such that

$$
\operatorname{tr}(\pi(F))=\left\{\begin{array}{l}
a(\psi) \text { for } \pi=i_{G M}(\psi \sigma), \quad \psi \in \Psi^{u}(M), \\
0 \quad \text { for } \pi=i_{G N}(\psi \tau), \quad \psi \in \Psi^{u}(N),
\end{array}\right.
$$

for any other standard Levi subgroup $N$ and a square-integrable modulo center represenrepresentation $\tau$ such that $\Theta_{t}(N, \tau) \neq \Theta_{t}(M, \sigma)$.

Proof. The proof of Proposition 3-4 is a generalization of ([8, 4.2, Proposition 1) where the proof of existence of pseudo-coefficients for semisimple $G$ is given based also on [3]. We consider only the case $M$ is a standard maximal Levi subgroup of $G$. The case of $M=G$ is about the construction of a specific pseudo-coefficient of $\sigma$. The proof is on the same lines but considerably easier. 
We remark that $\Psi^{u}(G)$ acts on $\Psi^{u}(M)$ in a usual way:

$$
\left.\psi \longmapsto \chi\right|_{M} \psi, \quad \chi \in \Psi^{u}(G), \quad \psi \in \Psi^{u}(M) .
$$

For $\psi \in \Psi^{u}(M)$, the stabilizer

$$
\operatorname{Stab}_{\Psi^{u}(G)}\left(i_{G M}(\psi \sigma)\right)
$$

is the group of all $\chi \in \Psi^{u}(G)$ such that

$$
\chi i_{G M}(\psi \sigma) \simeq i_{G M}(\psi \sigma) .
$$

We remind the reader that for all $\chi \in \Psi^{u}(G)$ we have

$$
\chi i_{G M}(\psi \sigma) \simeq i_{G M}\left(\left.\chi\right|_{M} \psi \sigma\right) .
$$

Lemma 3-5. Assume that $\chi \in \Psi^{u}(G)$ and $\psi \in \Psi^{u}(M)$. Then, for each irreducible constituent $\pi$ of $i_{G M}(\psi \sigma)$, the multiplicity of $\chi \pi$ in $\chi i_{G M}(\psi \sigma)$ is same as that of $\pi$ in $i_{G M}(\psi \sigma)$.

Proof. Obvious.

Lemma 3-6. Assume that for $\chi \in \Psi^{u}(G)$ and $\psi \in \Psi^{u}(M)$ there exists an irreducible constituent $\pi$ of $i_{G M}(\psi \sigma)$ such that $\chi \pi$ is an irreducible constituent of $i_{G M}(\psi \sigma)$. Then, $\chi \in S t a b_{\Psi^{u}(G)}\left(i_{G M}(\psi \sigma)\right)$. In particular, we have

$$
\operatorname{Stab}_{\Psi^{u}(G)}(\pi) \subset S t a b_{\Psi^{u}(G)}\left(i_{G M}(\psi \sigma)\right) .
$$

Proof. First, $\chi \pi$ is a common constituent of $i_{G M}(\psi \sigma)$ and $i_{G M}\left(\left.\chi\right|_{M} \psi \sigma\right)$. So, by Lemma 2-6, there exists $w \in W(M)$ such that

$$
\left.\chi\right|_{M} \psi \sigma=w(\psi \sigma) \text {. }
$$

Then, again by Lemma 2-6, we obtain

$$
\chi i_{G M}(\psi \sigma) \simeq i_{G M}\left(\left.\chi\right|_{M} \psi \sigma\right) \simeq i_{G M}(\psi \sigma) .
$$

Lemma 3-7. Let $\psi \in \Psi^{u}(M)$. Then, we have the following:

(i) If $\chi \in \operatorname{Stab}_{\Psi^{u}(G)}\left(i_{G M}(\psi \sigma)\right)$, then $a\left(\left.\chi\right|_{M} \psi\right)=a(\psi)$.

(ii) For each $\eta \in \Psi(G)$ and $\chi \in \operatorname{Stab}_{\Psi^{u}(G)}\left(i_{G M}(\psi \sigma)\right)$, we have

$$
a\left(\left.\left.\chi\right|_{M} \eta\right|_{M} \psi\right)=a\left(\left.\eta\right|_{M} \psi\right) .
$$

Proof. We prove (i). Since $\chi \in S t a b_{\Psi^{u}(G)}\left(i_{G M}(\psi \sigma)\right)$, we obtain

$$
i_{G M}\left(\left.\chi\right|_{M} \psi \sigma\right) \simeq \chi i_{G M}(\psi \sigma) \simeq i_{G M}(\psi \sigma) \text {. }
$$

So, by Lemma 2-6, there exists $w \in W(M)$ such that

$$
\left.\chi\right|_{M} \psi \sigma \simeq w(\psi \sigma) \simeq w(\psi) w(\sigma) .
$$


By definition of $W(D)$ (see (2-2) $)$, this implies $w \in W(D)$, and above relation can be written as follows:

$$
\left.\chi\right|_{M} \psi \sigma \simeq \psi_{w} w(\psi) \sigma
$$

where

$$
\psi_{w}=\left.w(\psi)^{-1} \chi\right|_{M} \psi
$$

Consequently, by the definition of the action of $W(D)$ on $D$ (see (2-3) ) we obtain

$$
\left.\chi\right|_{M} \psi \operatorname{Stab}_{\Psi(M)}(\sigma)=\psi_{w} w(\psi) \operatorname{Stab}_{\Psi(M)}(\sigma)=w \cdot \psi \operatorname{Stab}_{\Psi(M)}(\sigma) .
$$

This implies $a\left(\left.\chi\right|_{M} \psi\right)=a(\psi)$. This proves (i).

To prove (ii), we may assume that $\eta$ is unitary. Then, we obviously have

$$
\operatorname{Stab}_{\Psi^{u}(G)}\left(i_{G M}\left(\left.\eta\right|_{M} \psi \sigma\right)\right)=\operatorname{Stab}_{\Psi^{u}(G)}\left(i_{G M}(\psi \sigma)\right) .
$$

Now, the claim follows from (i).

Now, in order to complete the proof of Proposition 3-4, we apply ([3], Theorem 1.2). We define a $\mathbb{Z}$-linear form $f: R(G) \longrightarrow \mathbb{C}$ in several steps. We warn the reader that we use the same letter for a functional different than one from the statement of Theorem 1-1.

1. For each $\Psi^{u}(G)$-orbit $\mathcal{O}$ in $\Psi^{u}(M)$, we fix a representative $\psi_{\mathcal{O}} \in \mathcal{O}$ and an irreducible constituent $\pi_{\mathcal{O}}$ in $i_{G M}\left(\psi_{\mathcal{O}} \sigma\right)$. By Lemma 3-6, we have

$$
S t a b_{\Psi^{u}(G)}\left(\pi_{\mathcal{O}}\right) \subset S t a b_{\Psi^{u}(G)}\left(i_{G M}\left(\psi_{\mathcal{O}} \sigma\right)\right) .
$$

The quotient is finite and if $\chi$ ranges over representatives of the quotient, then $\chi \pi_{\mathcal{O}}$ ranges over the set of all mutually non-equivalent irreducible subrepresentations in $i_{G M}\left(\psi_{\mathcal{O}} \sigma\right)$ which are $\Psi^{u}(G)$-equivalent to $\pi_{\mathcal{O}}$. Any of those representations, have the same multiplicity in $i_{G M}\left(\psi_{\mathcal{O}} \sigma\right)$. Let $m_{\mathcal{O}}$ be the sum of their multiplicities. We define:

$$
f\left(\chi \pi_{\mathcal{O}}\right)=\frac{a\left(\psi_{\mathcal{O}}\right)}{m_{\mathcal{O}}}, \quad \chi \in S t a b_{\Psi^{u}(G)}\left(i_{G M}\left(\psi_{\mathcal{O}} \sigma\right)\right) .
$$

2. For each $\chi \in \Psi^{u}(G)$, we obviously have

$$
\operatorname{Stab}_{\Psi^{u}(G)}\left(\chi \pi_{\mathcal{O}}\right)=\operatorname{Stab}_{\Psi^{u}(G)}\left(\pi_{\mathcal{O}}\right)
$$

and

$$
S t a b_{\Psi^{u}(G)}\left(i_{G M}\left(\left.\chi\right|_{M} \psi_{\mathcal{O}} \sigma\right)\right)=\operatorname{Stab}_{\Psi^{u}(G)}\left(i_{G M}\left(\psi_{\mathcal{O}} \sigma\right)\right) .
$$

By, Lemma [3-5] and these remarks, the sum of multiplicities of $\Psi^{u}(G)$ equivalent representations of $\pi_{\mathcal{O}}$ which belong to $i_{G M}\left(\left.\chi\right|_{M} \psi_{\mathcal{O}} \sigma\right)$ is again $m_{\mathcal{O}}$. We let

$$
f\left(\chi \pi_{\mathcal{O}}\right)=\frac{a\left(\left.\chi\right|_{M} \psi_{\mathcal{O}}\right)}{m_{\mathcal{O}}}, \quad \chi \in \Psi^{u}(G)
$$

Lemma 3-7 (ii) shows that this is well-defined. 
3. For any other tempered irreducible representation (and, in particular, square-integrable modulo center representation) $\pi$ of $G$ we let

$$
f(\pi)=0 .
$$

4. For any quasi-tempered irreducible representation $\pi$ of $G$, we can write $\pi=\chi \pi^{u}$, where $\chi \in \Psi^{r}(G)$ and $\pi^{u}$ is tempered. We let

$$
f(\pi)=0,
$$

if $\pi^{u}$ is not in $\Psi^{u}(G) \pi_{\mathcal{O}}$ for any orbit $\mathcal{O}$ described in 1. But, if $\pi^{u} \in \Psi^{u}(G) \pi_{\mathcal{O}}$, for some $\mathcal{O}$, then we can write $\pi^{u}=\psi \pi_{\mathcal{O}}$, for some $\psi \in \Psi^{u}(G)$ uniquely determined modulo $\operatorname{Stab}_{\Psi^{u}(G)}\left(\pi_{\mathcal{O}}\right)$. We let

$$
f(\pi)=\frac{a\left(\left.\left.\chi\right|_{M} \psi\right|_{M} \psi_{\mathcal{O}}\right)}{m_{\mathcal{O}}} .
$$

Using (3-8) and Lemma 3-7 (ii) we see that this is well-defined.

6. Finally, we define $f$ on non-tempered Langlands quotients (see Lemma 2-11). let $f$ to be equal to zero on all standard modules induced from proper parabolic subgroups except in the following two obvious cases:

(a) The standard module $i_{G M}(\chi \psi \sigma)$, where $\chi \in \Psi(M)^{r,+}$ and $\psi \in$ $\Psi^{u}(M)$. In this case, we let

$$
f\left(i_{G M}(\chi \psi \sigma)\right)=a(\chi \psi) .
$$

(b) It is also possible that $\chi \in \Psi(M)^{r}$ belongs to the positive Weyl Chamber for the opposite parabolic $\bar{P}$ (see beginning of previous section). Then, there exists a unique standard maximal parabolic subgroup $Q$ with standard Levi $N$, and $w \in G$ such that $N=$ $w M w^{-1}$. Now, by ([3], Lemma 5.3 (iii)), we have

$$
i_{G M}(\chi \psi \sigma)=i_{G N}(w(\chi) w(\psi) w(\sigma)),
$$

in $R(G)$. Also, we have $w(\chi) \in \Psi(N)^{r,+}$. On the standard module $i_{G N}(w(\chi) w(\psi) w(\sigma))$ we let

$$
f\left(i_{G N}(w(\chi) w(\psi) w(\sigma))\right)=a(\chi \psi) .
$$

Thus, we have

$$
f\left(i_{G M}(\chi \psi \sigma)\right)=f\left(i_{G N}(w(\chi) w(\psi) w(\sigma))\right)=a(\chi \psi),
$$

for $\chi \in \Psi(M)^{r}$ such that $w(\chi) \in \Psi(N)^{r,+}$.

The third case is that $\chi \in \Psi(M)^{r}$ is in neither chamber. Then, $\chi \in \Psi(G)^{r}$, by standard description of unramified characters ([10], Section 2). In this case

$$
i_{G M}(\chi \psi \sigma)=\chi i_{G M}(\psi \sigma)
$$

is a quasi-tempered representation, and, by

$$
f\left(i_{G M}(\chi \psi \sigma)\right)=f\left(\chi i_{G M}(\psi \sigma)\right)=a(\chi \psi),
$$


by $1 .-4$.

This completes the construction of $\mathbb{Z}$-linear form $f: R(G) \longrightarrow \mathbb{C}$. In order to complete the proof of the proof of Proposition 3-4, we just need to check that it satisfies the assumptions of ([3], Theorem 1.2). First, let $N$ be a standard Levi subgroup of $G$ contained in $M$, and $\rho$ an irreducible supercuspidal representation of $N$ such that $\sigma$ is an irreducible subqoutient of $i_{M, N}(\rho)$. Then, by construction, $f$ is zero on irreducible representations which are not irreducible subqoutients of members of the family $i_{M, N}(\chi \rho)$ parameterized by $\chi \in \Psi(N)$. Then, as in the proof of Lemma 2-8, there exists an open compact subgroup $K$ such that $f$ is zero on all irreducible representations which does not have a non-zero $K$-invariant vector. This is (ii) in $([3,1.2)$. It remains to check (i) in $([3], 1.2)$. We need to check that for an arbitrary standard Levi subgroup $N$ of $G$ and an irreducible representation $\tau$ of $N$, the function $\chi \longmapsto f\left(i_{G, N}(\chi \tau)\right)$ is regular on $\Psi(N)$. By Lemma 2-1 applied to $N, \tau$ is a $\mathbb{Z}$-linear combination of standard modules for $N$. So, instead of being irreducible, we may assume that $\tau$ is a standard module for $N$ i.e.,

$$
\tau=i_{N N^{\prime}}\left(\chi^{\prime} \tau^{\prime}\right)
$$

$N^{\prime}$ is a standard Levi subgroup, $\tau^{\prime}$ is an irreducible tempered representation of $N^{\prime}$ and $\chi^{\prime} \in \Psi^{r,+}\left(N^{\prime}, N\right)$. Here, by definition $\Psi^{r,+}\left(N^{\prime}, N\right)$ is an analogue of $\Psi^{r,+}\left(N^{\prime}, G\right) \stackrel{\text { def }}{=} \Psi^{r,+}\left(N^{\prime}\right)$ defined in previous section. Now, by induction in stages, we have

$$
i_{G, N}(\chi \tau)=i_{G, N^{\prime}}\left(\left.\chi\right|_{N^{\prime}} \chi^{\prime} \tau^{\prime}\right) .
$$

We decompose $\chi=\chi^{r} \chi^{u}$ into its real $\chi^{r} \in \Psi^{r}(N)$ and unitary part $\chi^{u} \in$ $\Psi^{r}(N)$. Let $N^{\prime \prime}$ be a standard Levi subgroup such that $N^{\prime} \subset N^{\prime \prime} \subset N$ obtained by adjoining all simple roots orthogonal to $\left.\chi^{r}\right|_{N^{\prime}} \chi^{\prime}$ (see [10], Section 2 ). Then, $\left.\chi^{r}\right|_{N^{\prime}} \chi^{\prime}$ is an unramified character of $N^{\prime \prime}$ which is not orthogonal to any simple root that determines standard parabolic subgroup of $N^{\prime \prime}$. In particular, there exists $w \in G$ such that $N_{1}^{\prime \prime}=w N^{\prime \prime} w^{-1}$ is a standard Levi subgroup, and

$$
w\left(\left.\chi^{r}\right|_{N^{\prime}} \chi^{\prime}\right) \in \Psi^{r,+}\left(N_{1}^{\prime \prime}\right)
$$

(see for example [9], Section 1). Also, we can write

$$
i_{G, N^{\prime}}\left(\left.\chi\right|_{N^{\prime}} \chi^{\prime} \tau^{\prime}\right)=i_{G, N^{\prime \prime}}\left(\left.\chi^{r}\right|_{N^{\prime}} \chi^{\prime} i_{N^{\prime}, N^{\prime \prime}}\left(\left.\chi^{u}\right|_{N^{\prime}} \tau^{\prime}\right)\right) \text {. }
$$

Obviously, $i_{N^{\prime}, N^{\prime \prime}}\left(\left.\chi^{u}\right|_{N^{\prime}} \tau^{\prime}\right)$ is a direct sum of irreducible tempered representations, say $\tau^{\prime \prime}$ of $N^{\prime \prime}$. This implies that $i_{G, N^{\prime}}\left(\left.\chi\right|_{N^{\prime}} \chi^{\prime} \tau^{\prime}\right)$ is a direct sum induced representations:

$$
i_{G, N^{\prime \prime}}\left(\left.\chi\right|_{N^{\prime}} \chi^{\prime} \tau^{\prime \prime}\right)
$$

By above, in $R(G)$, we have

$$
i_{G, N^{\prime \prime}}\left(\left.\chi\right|_{N^{\prime}} \chi^{\prime} \tau^{\prime \prime}\right)=i_{G, N_{1}{ }^{\prime \prime}}\left(w\left(\left.\chi\right|_{N^{\prime}} \chi^{\prime}\right) w\left(\tau^{\prime \prime}\right)\right)
$$


But the last induced representation is a standard module. Now, by the construction of $f, f=0$ on all standard modules except those described in steps 1.-5. above. This means that we have one of the following two cases: a) $N^{\prime \prime}$ is conjugate to $G$. In this case $N_{1}^{\prime \prime}=N^{\prime \prime}=N^{\prime}=G, \tau^{\prime}$ is tempered irreducible representation of $G$, and $i_{G, N^{\prime}}\left(\left.\chi\right|_{N^{\prime}} \chi^{\prime} \tau^{\prime}\right)=\chi \chi^{\prime} \tau^{\prime}$. Thus, by the construction 1.-4., $\chi \longmapsto f\left(\chi \chi^{\prime} \tau^{\prime}\right)$ is regular.

b) $N^{\prime \prime}$ conjugate to $M$. In this case, $N^{\prime}=N^{\prime \prime}$, and $\tau^{\prime}$ must be conjugate to an element of the orbit $\Psi^{u}(M) \sigma$ (see 5. above). The discussion in 5. implies that $\chi \longmapsto f\left(i_{G, N^{\prime}}\left(\left.\chi\right|_{N^{\prime}} \chi^{\prime} \tau^{\prime}\right)\right)$ is regular.

This finally verifies (i) of $([3], 1.2)$, and completes the proof of the proposition.

\section{REFERENCES}

[1] J. Bernstein, RÉdigé PAR P. Deligne, Le 'centre' de Bernstein, Représentations des groupes réductif sur un corps local, Herman, Paris, 1984.

[2] J. Bernstein, Draft of: Representations of p-adic groups (lectures at Harvard University, 1992, written by Karl E. Rumelhart)

[3] J. N. Bernstein, P. Deligne, D. Kazhdan, Trace Paley-Wiener theorem for reductive p-adic groups, J. Analyse Math. 47 (1986), 180-192.

[4] J. Bernstein, A. V. Zelevinsky, Representations of the group $G L(n, F)$, where $F$ is a local non-Archimedean field (Russian), Uspehi Mat. Nauk 31 (1976), no. $3(189), 5-70$.

[5] I. N. Bernstein, A. V. Zelevinsky, Induced representations of reductive p-adic groups I, Ann. Sci. École Norm Sup., 10 (1977), 441-472.

[6] P. CARTiER, Representations of p-adic groups: a survey. Automorphic forms, representations and L-functions (Proc. Sympos. Pure Math., Oregon State Univ., Corvallis, Ore., 1977), Part 1, Proc. Sympos. Pure Math., XXXIII Amer. Math. Soc., Providence, R.I (1979), 111-155.

[7] W. Casselman, Introduction to the theory of admissible representations of $p$ adic reductive groups, preprint.

[8] L. Clozel, On limit multiplicities of discrete series representations in spaces of automorphic forms, Invent. Math. 85 (1986), 265-284.

[9] G. MuIć, Construction of Steinberg type representations for reductive p-adic groups, Math. Z. 253 (2006), no. 3, 635-652.

[10] G. Muić, A Geometric Construction of Intertwining Operators for Reductive p-adic Groups, Manuscripta Math. 125 (2) (2008), 241-272.

[11] D. RenARD, Représentations des groupes réductifs p-adiques. (French) [Representations of p-adic reductive groups] Cours Spcialisés [Specialized Courses], 17. Société Mathématique de France, Paris, 2010. vi+332 pp.

[12] F. Shahidi, A proof of Langlands conjecture on Plancherel measures; complementary series for p-adic groups, Annals of Math. 132 (1990), 273-330.

[13] J. L. Waldspurger, La formule de Plancherel pour les groupes p-adiques, d'après Harish-Chandra, Journal of the Inst. of Math. Jussieu 2(2), 235-333, 2003.

Department of Mathematics, Faculty of Science, University of Zagreb, BiJENIČKA 30, 10000 ZAGREB, CROATIA

E-mail address: gmuic@math.hr 